\title{
TUNNELING IN SEMICONDUCTOR NANOSTRUCTURES: PHYSICS AND DEVICES
}

\author{
H. LÜTII \\ Institut für Schicht- und Ionentechnik (ISI), Forschungszentrum Jülich GmbII \\ 52425 Jülich, Germany
}

\begin{abstract}
Since modern semiconductor epitaxy techniques have reached the present level of perfectness, resonant tunneling through double barrier structures has attracted new interest, also with respect to novel device applications. The present review shortly discusses the underlying physical concepts of resonant tunneling through semiconductor multiple heterostructures. Beside resonant tunneling diodes also concepts for tunneling transistors are discussed. In particular, a novel $2 \mathrm{D}-2 \mathrm{D}$ tunnel transistor is presented, in which tunneling between two ncighboring two-dimensional electron gases yields current-voltage characteristics with two types of instabilities depending on the applied gate voltage. Scaling down the lateral dimensions of a resonant tunneling diode leads to quantum boxes or dots, in which the effect of single electron tunneling with Coulomb-blockade can be observed. The underlying physics is discussed on the basis of recent experiments and theoretical calculations. Finally some possible future applications both of resonant tunneling diodes and of single electron devices are presented.
\end{abstract}

PACS numbers: 72.80.Ey, 73.40.Gk, 85.30.Mn

\section{Introduction}

Since semiconductor epitaxy techniques such as molecular beam epitaxy (MBE), metalorganic vapor phase epitaxy (MOVPE) and metalorganic MBE (MOMBE) have reached an advanced state of perfection, such that III-V and $\mathrm{Si} / \mathrm{SiGe}$ multiple heterostructures can be fabricated with vertical layer dimensions on the 0.1-10 nanometer scale and an interface sharpness of 1 to 3 atomic layers, tunneling of carriers through such structures has attracted much interest both for fundamental rescarch and for application reasons. In this respect resonant tunneling devices (diodes and transistors) are the only quantum devices so far which function at room temperature. Because of their highly nonlinear current voltage $(I-V)$ characteristics these devices are attractive as multifunctional components which provide more complex logic functions with less circuit complexity.

Additional lateral structuring of such tunneling structures leads to 3-dimensional nanostructures, the so-called quantum dots or boxes in which the electronelectron interaction becomes directly observable as the so-called single-electron-tunneling (SET) effect [1]. SET in these nanostructures thus provides new path 
ways for the study of electron-electron interaction but also leads to novel computer concepts called quantum cellular automata [2]. The present review provides a short overview over some essential underlying physical concepts of these tunneling nanostructures of III-V semiconductor alloy systems and mentions, in a preliminary way, some possible applications in future micro (nano) electronics.

\section{Resonant tunneling diodes}

Resonant tunneling devices, in their simplest form resonant tunneling diodes (RTDs), are fabricated as multiple heterostructure layer systems, where a material with larger band gap (e.g. AlGaAs) forms two thin barriers (thickness approximately $2 \mathrm{~nm}$ ), about $5 \mathrm{~nm}$ apart from each other, within a semiconductor with smaller band gap (e.g. GaAs). The device is fabricated as a lithographically structured mesa structure with two ohmic contacts; alternatively the device might be isolated from the surrounding by ion implantation. Biasing the diode between top and bottom contacts causes current flow. For conduction electrons, which propagate wave-like, the two barriers can be penetrated by tunneling; they act as semitransparent mirrors for the electron waves similarly as mirrors do for light waves in a Fabry-Perrot interferometer. When multiples of half the electron wavelength $(n \lambda / 2)$ match the distance between the two inner sides of the barriers (well region), the transparency of the double barrier structure reaches high values (in the ideal case one) due to the so-called resonance tunneling [3]. A convenient bias between the two contacts rises the energy of electrons on the emitter side such that electrons near the Fermi energy $E_{\mathrm{F}}$ fulfill the matching condition $d=\lambda / 2$ and a strong current through the RTD results. A further increase in the applied voltage brings the electrons on the emitter side out of resonance, the current breaks down; but it rises again when the effect of the second barrier becomes negligible. The current voltage $(I-V)$ characteristic of a RTD is $\mathrm{N}$ shaped and exhibits a negative differential resistance (NDR) (Fig. 1). This highly nonlinear $I-V$ characteristic with NDR is the basis for the application interest in resonance tunneling devices.

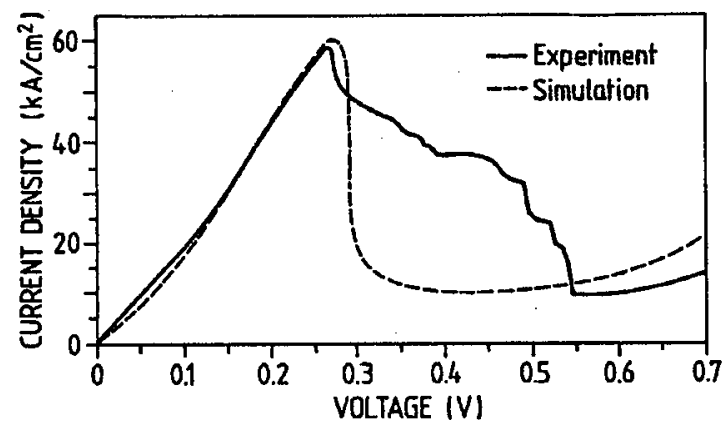

Fig. 1. Measured (full line) and calculated (broken line) current-voltage (I-V) characteristic of an AlAs double barrier (barrier thickness: 6 monolayers) RTD embedded in $\mathrm{In}_{0.17} \mathrm{Ga}_{0.83} \mathrm{As}$; well width $5 \mathrm{~nm}$, diode mesa area $4 \mu \mathrm{m} \times 4 \mu \mathrm{m}$ (after [5]). 
Apart from oscillators and rectifiers the bistability of the characteristics can be used in logic circuits, flip-flop memories etc. In this respect RTD devices have a considerable potential for a reduction in circuit complexity. With much less single devices and lower circuit complexity considerably higher logic complexity can be achieved. Faster and more complex circuits can be designed with significantly relaxed lithographic requirements for lateral structuring.

\section{Coherent and sequential tunneling}

The quantum mechanical transmission of an electron wave through the double barrier structure is described mathematically by a coherent solution of the Schrödinger equation outside the barrier regions (left and right) and in between the two barriers, in the well $[4,5]$. Due to the thin barrier regions (couple of monolayers) the quantized states in the well "leak out" through the barriers and couple colherently to moving plane wave states, left and right. As a result the states in the well can be considered as metastable and the lifetime of the electron within the well is finite. The corresponding quasi-bound states between the barriers are therefore described apart from their quantum energy $E_{\mathbf{i}}$, by a lifetime $\tau$ during which a tunneling electron occupies this state. Through the uncertainty relation $\tau \Gamma_{0}=\hbar$ the lifetime is related to the energetic half width $\Gamma_{0}$ of the quasi-bound state. For typical AlAs/GaAs RTDs with barrier widths of 6 monolayers $(6 \mathrm{ML}=$ $1.7 \mathrm{~nm}$ ) AlAs and a GaAs well width of $5 \mathrm{~nm}$ typical half widths $\Gamma_{0}$ are $2 \mathrm{meV}$ and $25 \mathrm{meV}$ for the first and second quasi-bound state, respectively.

If one calculates the tunneling current solely based on the described coherent tunneling [5], the simulated $I-V$ curves exhibit a much too sharp resonance peak and the peak-to-valley ratio $(P V R)$ exceeds measured data by more than a factor of 100 . The reason is found in inelastic scattering events within the double barrier region which lead to sequential transmission of electrons through the barriers. A coherent wave function does no longer exist over the whole RTD. Without accounting for details of the scattering mechanism one can formally describe the combination of resonant tunneling and sequential or incoherent tunneling (inelastic scattering) by a generalization of the Lorentzian form of resonant transmission based on the Breit-Wigner formalism. For a symmetric RTD and an electron with energy $E$ Stone and Lee [6] derive a total transmission probability in the presence of inelastic scattering as

$$
T_{\text {tot }}=\frac{(1 / 4) \Gamma_{0} \Gamma}{\left(E-E_{r}\right)^{2}+(1 / 4) \Gamma^{2}}
$$

where $E_{r}$ is the energy of the quasi-bound state, and $\Gamma_{0}$ the inherent half width of the quasi-bound eigenstate (energy $E_{r}$ ) in purely coherent transmission. $\Gamma=$ $\Gamma_{0}+\Gamma_{\mathrm{i}}$ is the total half width of the resonance which is obtained by folding the resonance peak by a Lorentzian with half width $\Gamma_{\mathrm{i}}$, which describes the inelastic scattering probability. Within this formalism inelastic scattering, i.e. incoherent or sequential tunneling diminishes the $P V R$ according to

$$
P V R=(P V R)_{0} \frac{\Gamma_{0}}{\Gamma_{0}+\Gamma_{\mathrm{i}}},
$$

where $(P V R)_{0}$ is the peak-to-valley ratio as calculated without scattering. According to Fig. 1 these simulations allow a satisfying formal description of measured 
$I-V$ curves, even though there is no direct physical explanation of the scattering mechanisms.

A detailed theoretical study of various scattering effects within RTDs, including acoustic and optical phonon, impurity, alloy disorder and interface roughness scattering, has been given by Chevoir and Vinter [7]. For RTDs prepared from a combination of a direct and an indirect semiconductor as $\mathrm{AlAs} / \mathrm{GaAs} \Gamma$ to $X$ valley scattering plays an important role. In AlAs/GaAs diodes, e.g., AlAs acts as a potential barrier for $\Gamma$ electrons but as a potential well for $X$ electrons. $\Gamma$ electrons from the supply (emitter) region of the RTD might therefore tunnel through $X$ states in the barriers if they gain $k$-vector (from $\Gamma$ to $X$ ) due to a scattering event. The relevance of $\Gamma$ to $X$ valley scattering for sequential tunneling has been shown from the pressure dependence of the $I-V$ curves of RTDs by Mendez et al. [8] and Brugger et al. [9]. The effect of different types of interface roughness on interface scattering has been demonstrated by Förster et al. [10].

\section{High frequency behavior}

The important figure of merit of a RTD is the peak-to-valley ratio $I_{\text {peak }} / I_{\text {valley }}$, which defines the extension of the negative differential resistance. For the use in $\mathrm{RF}$ circuits the persistence of the NDR up to highest frequencies is necessary: one defines a maximum cut-off frequency $f_{\max }$, up to which NDR, i.e. a non-negligible $P V R$ is observed. The simplest equivalent circuit for a RTD which one would expect, must at least consist of a resistance $R_{\mathrm{d}}\left(-\left|R_{\mathrm{d}}\right|\right.$ because of NDR) in parallel with a diode capacitance $C_{\mathrm{d}}$ and again in series with a series resistance $R_{\mathrm{s}}$, mainly due to contacts. This simple equivalent circuit describes indeed the RF behavior up to frequencies of $26 \mathrm{GIIz}$ [11]. As is obvious from Fig. 2 the complex impedance $Z$ of an AlAs/GaAs RTD as determined by a four-port scattering $(S)$ parameter measurement is well described by a simulation using the simple RTD equivalent

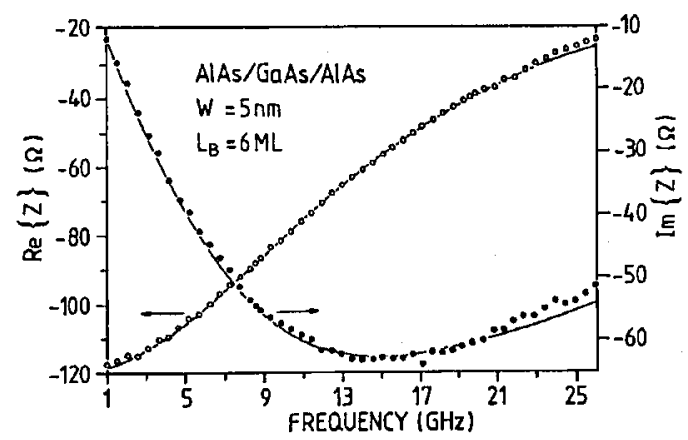

Fig. 2. Real and imaginary part of the complex impedance $Z$ of an AlAs/GaAs/AlAs double barrier RTD (well width $5 \mathrm{~nm}$, barrier thickness: 6 monolayers), measured by an $S$-parameter set-up. The full lines through the data points are a fit based on a simple equivalent circuit (see text) (after [11]). 
circuit consisting of the three parameters $R_{\mathrm{d}}, C_{\mathrm{d}}, R_{\mathrm{s}}$. Based on this circuit the maximum cut-off frequency is obtained as

$$
f_{\max }=\frac{1}{2 \pi\left|R_{\mathrm{d}} C_{\mathrm{d}}\right|} \sqrt{\frac{-R_{\mathrm{d}}}{R_{\mathrm{s}}}-1} .
$$

From the simulation (Fig. 2) one derives $f_{\max }$ values between 65 and $110 \mathrm{GHz}$, depending on the particular operating point, for a diode with a mesa area of $4 \times 4 \mu \mathrm{m}^{2}$ and a $P V R$ of 4.3 at $300 \mathrm{~K}$. Scaling down the mesa area by actor of 10 , therefore, should easily lead to $f_{\max }$ values of a couple of hundred $\mathrm{GHz}$, of course at lower current and power values.

\section{Tunnel transistors}

RTDs are two-port devices. In an integrated circuit additionally amplifying three-port devices, transistors, are necessary. More compact circuits could be built by devices in which the transistor function is integrated with the RTD function within one and the same device. Several types of resonant tunneling transistors (RTT) have been suggested and fabricated. One approach consists in incorporating a double barrier diode into the emitter-base barrier of a unipolar hot electron transistor (IIET). This resonant hot electron transistor (RHET) exhibits a non-monotonic form of the $I-V$ characteristics thus enabling a multiple state transistor. Realised in the GaAs/AlGaAs material system [12] the device suffered from a poor current gain of 3 to 4 and a poor $P V R$ in the transfer characteristics. In the InGaAs/In(GaAl)As system, where higher and thinner barriers are possible and intervalley scattering can be reduced, the peak current densities are increased by a factor of about four, the current gain rises to approx. 17 and a $P V R$ of about 10 can be achieved [13]. Recent redesigned RHETs were able to achieve cut-off frequencies of about $120 \mathrm{GHz}$ [14].

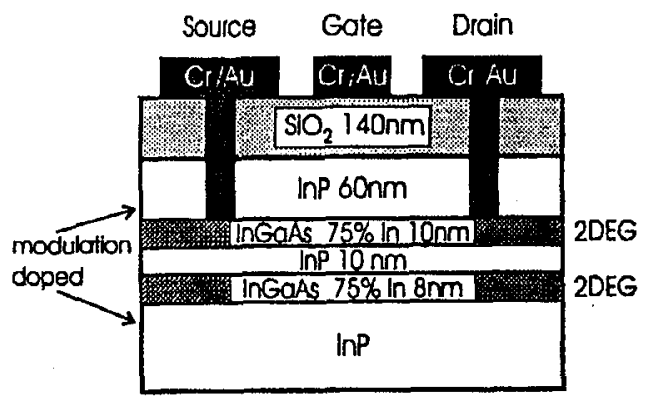

Fig. 3. Scheme of a 2D-2D tunnel transistor (Tunnel-HEMT), where tunneling between two two-dimensional electron gases (2DEG) is controlled by a gate [15].

A conceptually new approach is the two-dimensional to two-dimensional (2D-2D) tunnel transistor first proposed by Leuther et al. [15]. According to Fig. 3 two modulation doped $\operatorname{In}_{0.75} \mathrm{Ga}_{0.25}$ As channels of different thickness (different subband energies) are separated by a $10 \mathrm{~nm}$ thick InP barrier, through which tunneling 
is possible between the two two-dimensional electron gases (2DEGs) in the channels. The electron concentration within both 2DEGs is controlled by a metallic gate, isolated by a $\mathrm{SiO}_{2}$ layer. The upper channel is contacted by non-alloyed metallic source and drain contacts which are filled in into lithographically opened holes. This type of ohmic contact is possible only to InGaAs channels, where Fermi-level pinning occurs in the conduction band for sufficiently ligh In concentrations. The device structure in Fig. 3 can be considered as a HEMT (called: tunnel (T) IIEMT), where a second 2DEG channel is coupled by tunneling to the normal upper channel. Compared with other RTT structures there are several advantages: Tunneling can be easily controlled by a surface gate since there is essentially no screening between the channels and the gate. In contrast to standard RTDs, where tunneling occurs between 3D-2D-3D regions, tunneling in the T-HEMT is a 2D-2D process. The condition for a tunneling process between the two 2D states is the conservation of the total energy and of the in-plane momentum. Given the parabolic energy dispersion in the 2DEGs tunneling occurs only for one particular fixed voltage across the barriers.

Depending on the height of the voltage drop resonance occurs between both ground states in the channels or if the first subband-level in one channel energetically equals the ground state of the other one. Depending on the height of the gate voltage $V_{\mathrm{G}}$ two types of $I-V$ characteristics (source-drain current $I_{\mathrm{DS}}$ versus source-drain voltage $V_{D S}$ ) are found for the T-IIEMT (Fig. 4). For lower $V_{G}(-8$ to $-10 \mathrm{~V}$ ) there is a sudden increase in the drain current. This increase shifts to higher $V_{\mathrm{DS}}$ with lower gate voltages (Fig. 4, top). Upon reversing the source-drain voltage a strong hysteresis appears, which has not been completely understood so far. For higher gate voltages $V_{\mathrm{G}}(-13.2$ to $13.8 \mathrm{~V})$ there is a distinct NDR with a $P V R$ of up to 3.7 at $4 \mathrm{~K}$. The onset of the NDR shifts to higher drain voltages $V_{\mathrm{DS}}$ for increasing gate voltage $V_{\mathrm{G}}$.

The performance of the T-IIEMT has been roughly understood as follows: A finite gate voltage separates the source and drain region by depletion below the gate spatially from each other, such that two separate tunnel resistances below the drain and below the source region have to be considered. They connect the two 2DEG channels with each other. In the case of low gate voltages the 2DEG channel resistances are of the same order and considerably lower than the tunnel resistance across the InP barrier. Therefore the current $I_{\mathrm{DS}}$ only flows through the upper channel. At a particular higher source-drain voltage $V_{\mathrm{DS}}$ the tunnel diodes come into resonance and the current $I_{\mathrm{DS}}$ starts to flow through the lower channel, too: a steep increase in $I_{\mathrm{DS}}$ results (Fig. 4, top). The shift of this step with increasing $V_{\mathrm{G}}$ can be understood by the corresponding variation of the voltage drops across the different resistances. At higher gate voltages (Fig. 4, bottom) the upper channel is completely depleted and the current $I_{\mathrm{DS}}$ flows through the second 2DEG channel only and the two tunnel resistances in series. In this case the $I-V$ characteristic is similar to that of a normal RTD. With higher gate voltages the resistance of the lower channel increases, which leads to a lower voltage drop at the tunnel diodes. A higher $V_{\mathrm{DS}}$ is required to bring the T-HEMT into resonance. This explains the shift of the NDR region with varying $V_{\mathrm{G}}$. This qualitative interpretation of the $I-V$ characteristics can be made quantitatively at least for $\mathrm{dc}$ performance using 


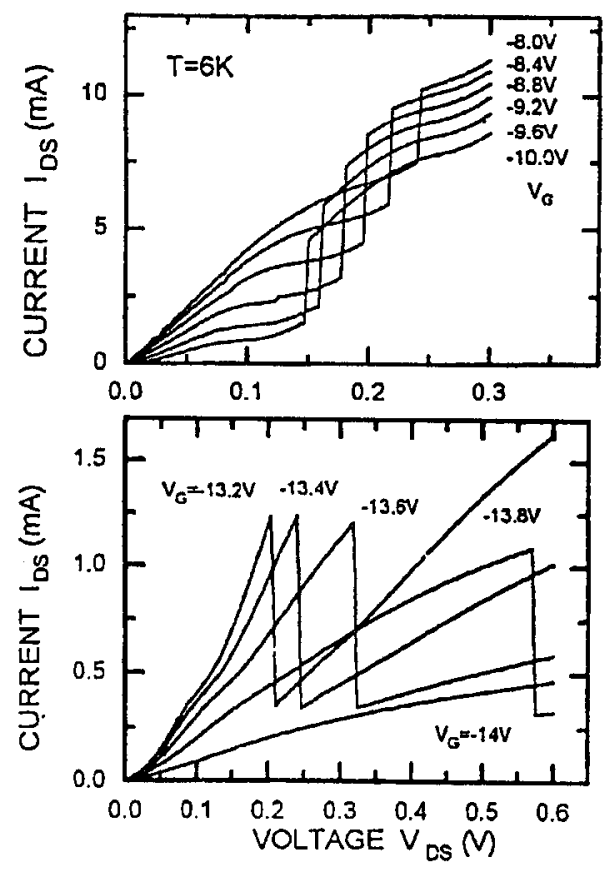

Fig. 4. Drain-source current-voltage $\left(I_{\mathrm{DS}}-V_{\mathrm{DS}}\right)$ characteristic of the $2 \mathrm{D}-2 \mathrm{D}$ tunnel transistor in the InGaAs/InP material system (see Fig. 3), for different gate voltages $V_{\mathrm{G}}$, measured at $6 \mathrm{~K}[15]$. Upper part: gate voltage regime below $-10 \mathrm{~V}$; lower part: gate voltage regime above $-13 \mathrm{~V}$.

a selfconsistently calculated band scheme for the tunneling process [15]. A deeper understanding of the T-IIEMT performance requires a detailed stability analysis of the whole complex system which has not been made so far. The presented T-IIEMT data result from recent investigations of this novel device concept. An improved technology based on experience with IIEMTs will certainly bring further progress. In particular, more sophisticated layer structures enabling higher tunnel barriers and better Schottky gates will allow the room temperature performance of the T-HEMT and also the gate control by considerably lower gate voltages.

\section{Single electron tunneling}

When the lateral mesa dimensions of RTDs are decreased sufficiently, lateral confinement of the carriers becomes significant in addition to the vertical confinement due to the built-in tunneling barriers. For AlGaAs/GaAs RTDs lateral dimensions of approximately 100 nanometers cause a confinement potential, which is much more restricted laterally, i.e. parallel to the heterostructure interfaces, since depletion space charge layers with a depth of about $50 \mathrm{~nm}$ have to be taken into account at the etched side walls of the mesas. Electrons tunneling into 
such a 3D-confined area, a quantum box, are "squeezed" together and "feel" a strong Coulomb interaction, when more than one electron occupies the quantized energy states within the confined spatial region between the two AlGaAs barriers. The quantized energy levels $\varepsilon$ within the box shift to higher energies $\varepsilon_{1}, \varepsilon_{2}, \varepsilon_{3}, \ldots$ each time, when a further electron tunnels from the emitter $(E)$ side into the box. Depending on the occupation of such a level with one, two or three electrons the resonance condition, that the level coincides energetically with the Fermi energy $\mu_{\mathrm{E}}$ (or $E_{\mathrm{F}}$ ) on the emitter side, appears at distinct increasing applied voltages (Fig. 5a). The tunneling current through the box increases stepwise, each time when a further electron tunnels through the first barrier (Fig. 5b). A step-like $I-V$ characteristic is measured [16] for small biases as compared with those in the NDR regime in the normal N-shaped characteristic of a RTD (Fig. 5b, inset). This SET effect is superimposed on the size quantization, i.e. the level splitting due to 3D confinement. Even if lateral confinement is low and the energy levels due to lateral quantization are close to each other or nearly degenerate, the steps due to SET, i.e. Coulomb interaction of the electrons within the box might be visible at sufficiently low temperature. In the experimental example of Fig. 5a temperature of $23 \mathrm{meV}$ was used to observe the effect because the energy separation between the different single electron levels $\varepsilon_{i}$ is in the order of a couple of meV [16].

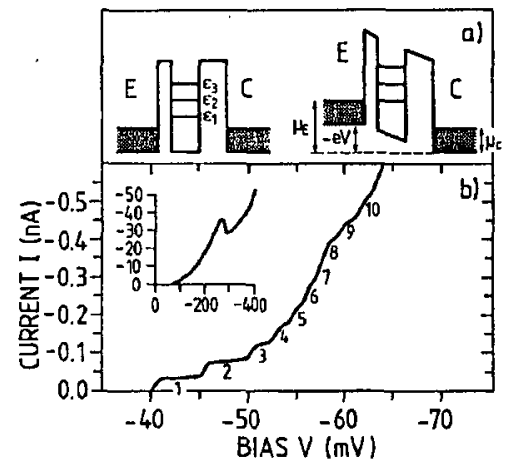

Fig. 5. Single electron tunneling through an $\mathrm{Al}_{0.3} \mathrm{Ga}_{0.7} \mathrm{As} / \mathrm{GaAs}$ resonant tunneling diode; dimensions of the diode: mesa diameter $350 \mathrm{~nm}$, 1st barrier thickness: $5 \mathrm{~nm}$, 2nd barrier thickness: $9 \mathrm{~nm}$, well width: $10 \mathrm{~nm}$; (a) Conduction band scheme without applied voltage (left) and with bias (right), $E$ emitter, $C$ collector, $\varepsilon_{i}$ single electron energy levels, $\mu_{E}$ and $\mu_{C}$ electrochemical potential on emitter and collector side; (b) Current-voltage $(I-V)$ characteristic measured at a temperature $T=23 \mathrm{mK}$. The numbers at the curve indicate tunneling of $1,2,3, \ldots$ single electrons. Inset: $I-V$ curve measured over an extended bias range [16].

In a recent theoretical paper Indlekofer et al. [17] used real-time Green's-function theory to describe nonequilibrium transport in the single-electron regime through a laterally confined quantum well coupled to electron reservoirs. Material parameters were taken for the AlGaAs/GaAs system as in the experiment of Fig. 5. Multiple subbands, electron spin, intrawell scattering and Coulomb inter- 
action were included. As is expected from simple considerations the detailed shape of the $I-V$ characteristic depends on the transparency of the two barriers: equal barriers (i.e. equal transparency), or high and low transparency at the emitter and collector side, respectively. The latter two cases mean strong or weak charging condition within the box. The calculation shows that under weak charging conditions the expectation value "particle number" in the box as an average value does not have integer values. The case of strong charging condition also realized in the experiments of Fig. 5 implies a thinner barrier at the emitter side $(5 \mathrm{~nm})$ and a thicker, less transparent one $(9 \mathrm{~nm})$ at the collector side. The tunneling electrons thus spend most of the time inside the box (well width $10 \mathrm{~nm}$ ), and we consequently expect the electron number to be nearly an integer. This is indeed seen in Fig. 6b (broken line). The theoretical results of Fig. 6a, where Coulomb interaction between the tunneling electrons is not taken into account, only show steps due to quantum confinement. The steps due to Coulomb interaction, i.e. SET are missing. The steps at 2, 6 and 12 in electron number correspond to the degeneracy of the assumed harmonic oscillator levels, including spin. For the case of SET including Coulomb interaction (Fig. 6b) the width of the first plateau, in comparison to Fig. 6a, shows the Coulomb interaction: The second electron tunneling into the box must overcome the repulsion. The electron number in Fig. $6 \mathrm{~b}$ increases at most by 1 from one step to the next one in spite of degeneracies in the well states.
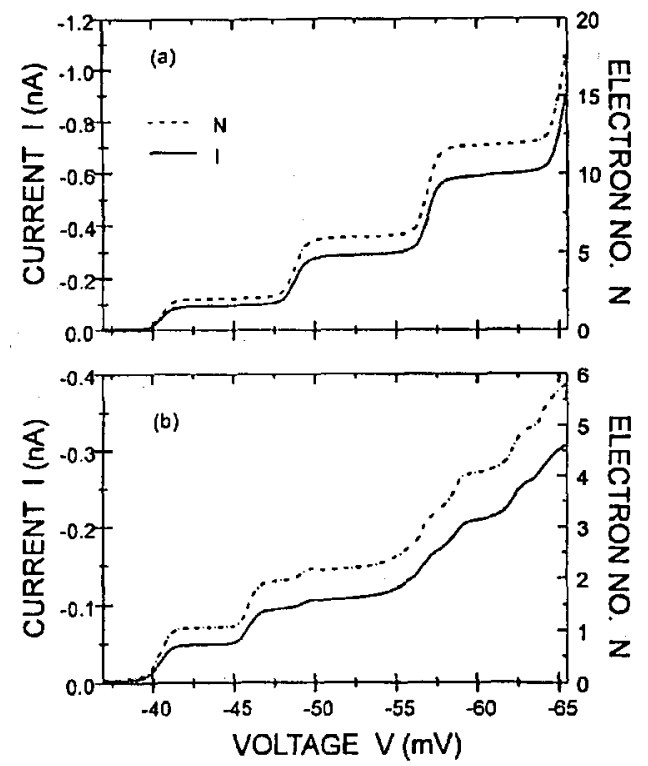

Fig. 6. Calculated tunneling current $I$ (full line) through and electron number $N$ within a quantum box (broken line) as a function of bias voltage $V$. The quantum box is formed by two $\mathrm{Al}_{0.3} \mathrm{Ga}_{0.7}$ As barriers with thickness of $5 \mathrm{~nm}$ (1st barrier) and $9 \mathrm{~nm}$ (2nd. barrier), i.e. strong charging condition, embedded in GaAs. (a) Coulomb interaction between electrons in the well neglected; (b) Coulomb interaction within the well induces additional steps (SET) [17]. 
The current step height of about $50 \mathrm{pA}$ agrees well with the experimental data in Fig. 5b.

The calculation of the SET effect [17] furthermore shows that the generally accepted description of the energy separation between the $N$-th and $(N+1)$-st level in terms of a quantum box capacity $C$

$$
\varepsilon_{N+1}-\varepsilon_{N}=\frac{\varepsilon^{2}}{C}
$$

is not accurate at least for low occupation within the dots. Nevertheless one might use Eq. (4) as a rough estimate for SET effects, at least their dependence on dimension parameters. Scaling down the dimensions of an RTD, in particular the mesa area, therefore increases the quantum level separation and finally should allow the observation of SET at higher temperatures. Quantum boxes and SET might promise an eventual future quantum electronics at room temperature or slightly below. Estimations lead to required box dimensions in the order of 5 to $10 \mathrm{~nm}$.

\section{Applications}

Since resonant tunneling transistors are in a preliminary state of development, applications of tunneling devices are known so far mainly for RTDs. The application interest in RTDs is based on the N-shaped $I-V$ characteristics which yields depending on the operating points regimes of stability, instability and bistability. As a detailed analysis of the corresponding differential equations shows, the regimes of instability with selfgeneration of oscillations, of stability and bistability sensitively depend on the circuit parameters diode capacity, series resistance and inductance [5]. Using the instability in the NDR regime high speed oscillators for frequencies up to several hundred GIIz have been built [18]. By integrating a number of RTDs either in parallel [19] or in series [20] a structure with multiple peaks in the $I-V$ characteristics can be made. Such devices, with multiple stable operating points, have been shown to be capable of a number of interesting circuit applications, such as frequency multiplier and parity generator. Lakhani et al. [21] have demonstrated an 11-bit parity generator using a single device with 5 RTD structures integrated vertically, replacing ten conventional exclusive "OR" gates. This example shows an interesting feature of resonant tunneling devices in general, namely to reduce circuit complexity but simultaneously enhance the logic complexity and ability of the circuit. A number of further applications have been discussed by Capasso et al. [22] and Luryi [23].

Applications of quantum boxes and SET are even more remote from present microelectronics. Severe technological problems have to be solved in order to fabricate these $3 \mathrm{D}$ nanostructures on large scale in a well defined way to enable a quantum electronics functioning at elevated temperatures, maybe around $300 \mathrm{~K}$. - As has been shown, a quantum box coupled to two reservoirs allows counting of single electrons from its tunneling $I-V$ characteristic. Single electrons thus can be used in principle to store and transfer bits of information.

Based on quantum dots and SET Lent et al. [2] have designed on purely theoretical grounds a new computer concept, called quantum cellular automation. The logic processor or memory consists of cells arranged laterally in close vicinity 

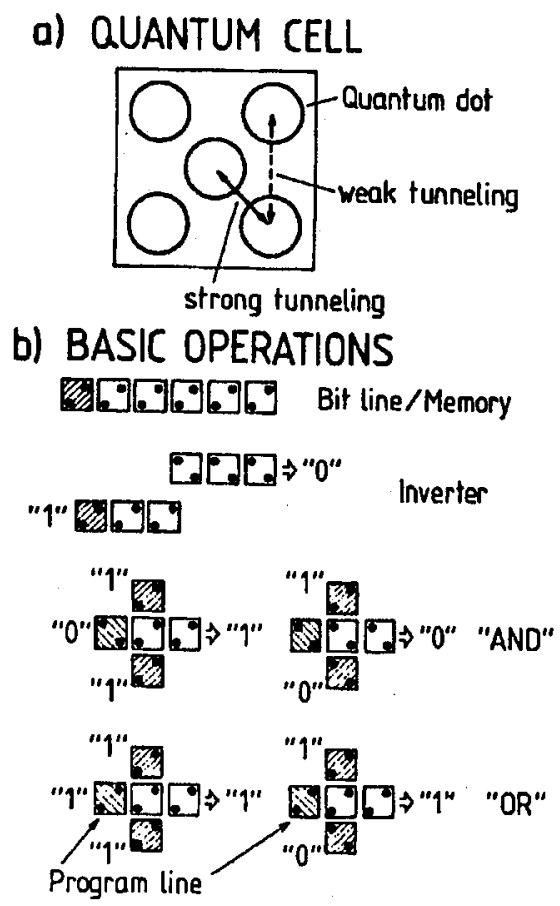

Fig. 7. Scheme of a quantum cellular automation, a novel theoretical concept of a quantum dot based computer (after [2]). (a) Quantum cell consisting of five quantum dots as the basic unit of the computer architecture: two electrons occupy the quantum states within the dots, they can tunnel through the central dot and form two stable orthogonal two-electron states. (b) Different arrangements of quantum cells for the realization of a bit line, a memory, an inverter and "AND" and "OR" gates. Two electrons as dark dots are plotted which indicate the initial electron configuration which initiates the information processing from left to right. The "AND" and "OR" gates also contain a program line which switches between "AND" and "OR" function.

(Fig. 7a). The distance between these dots allows tunneling between the center and the edge dots. A solution of the Schrödinger equation then shows that two single electrons filled into the quantum cell can assume two stationary quantum states $\pm P$ with the two electrons at the largest distance from each other, occupying the two outer quantum dots at the edges on two perpendicular diagonals. A slight perturbation by Coulomb interaction with electrons in a neighboring quantum cell then determines the realization of the eigenstate $+P$ or $-P$. The states $+P$ and $-P$ can represent a bit of information. Convenient lateral arrangements of the quantum cells with respect to each other then allow the realization of bit lines, memories, logic inverters as well as "AND" and "OR" gates (Fig. 7b). A major problem with these and other similar concepts of nanoelectronic circuits is the read-in and read-out of information. So far only tunneling microscopes (STM) can 
be applied for this purpose, but a solution for large scale production is not in sight.

Based on SET transistor structures, where the potential of quantum dots and therefore the tunneling in and out of these dots can be controlled by a gate electrode, a complete set of complementary logic circuits has been constructed and analyzed theoretically $[24,25]$. The implementation of simple logic and memory circuits might become possible which operate at temperatures between 20 and $70 \mathrm{~K}$. These fast low power consumption circuits might be coupled to standard $\mathrm{Si}$ CMOS circuits if compatible fabrication becomes possible on $\mathrm{Si}$ wafers.

\section{Conclusions}

As multifunctional devices resonant tunneling diodes and transistors certainly have a great potential for future high frequency electronics, when quantum effects severely limit further miniaturization in "main-stream" CMOS technology. But beside the development of novel device concepts based on resonant tunneling also new ways of circuit design must go hand in hand with the research on single devices in order to gain the full benefit of multifunctionality. This is in particular true, if single electron devices with tunneling in and out of quantum dots are used to store and handle bits of information by single electrons. Beside new concepts of computer architecture also convenient process technologies have to be developed to fabricate arrays of quantum dots and wires on the nanometer scale with a high degree of reliability.

\section{References}

[1] K.K. Likharev, A.B. Zorin, J. Low Temp. Phys. 59, 347 (1985).

[2] C.S. Lent, P.D. Tougaw, W. Porod, G. Bernstein, Nanotechnology 4, 49 (1993).

[3] C. Weisbuch, B. Vinter, Quantum Semiconductor Structures - Fundamentals and Applications, Academic Press Inc., Boston, New York, London 1991.

[4] B. Ricco, M. Ya Azbel, Phys. Rev. B 29, 1970 (1984).

[5] A. Förster, in: Festkörperprobleme/Advances in Solid State Physics, Ed. R. Helbig, Vol. 33, Vieweg, Braunschweig, Wiesbaden 1994, p. 37.

[6] A.D. Stone, P.A. Lee, Phys. Rev. Lett. 54, 1196 (1985).

[7] F. Chevoir, B. Vinter, Appl. Phys. Lelt. 55, 1859 (1989).

[8] E.E. Mendez, W.I. Wang, E. Calleja, C.E.T. Goncalves da Sil, Appl. Phys. Lett. 50, 1263 (1987).

[9] H. Brugger, U. Meiners, C. Wölk, R. Deufel, A. Marten, M. Rossmanith, K. v. Klitzing, R. Sauer, in: Proc. 21st European Solid State Device Research Conf., ESSDERC, Lausanne, Microelectron. Eng. 15, 663 (1991).

[10] A. Förster, J. Lange, D. Gerthsen, Ch. Dieker, H. Lüth, J. Phys. D, Appl. Phys. 27, 175 (1994).

[11] Th. Scheuermann, Ph.D. Thesis at RWTH, Aachen 1994.

[12] N. Yokoyama, K. Imamura, Electron. Lett. 22, 1228 (1986).

[13] N. Yokoyama, S. Muto, H. Ohnishi, K. Imamura, T. Mori, T. Inata, in: Physics of Quantum Electron Devices, Ed. F. Capasso, Springer, Berlin 1990, p. 253.

[14] T. Mori, T. Adachihara, M. Takatsu, H. Ohnishi, K. Imamura, S. Muto, N. Yokoyama, Electron. Lett. 27, 1523 (1991). 
[15] A. Leuther, M. Hollfelder, H. Hardtdegen, H. Lüth, to be published in 1996.

[16] T. Schmidt, M. Tewordt, R.H. Blick, R.J. Haug, D. Pfannkuche, K. v. Klitzing, A. Förster, H. Lüth, Phys. Rev B 51, 5570 (1995).

[17] M. Indlekofer, J. Lange, A. Förster, H. Lüth, Phys. Rev. B 53, 7392 (1996).

[18] E.R. Brown, T.C.L.G. Sollner, C.D. Parker, W.D. Goddhue, C.L. Chen, Appl. Phys. Lett. 55, 1777 (1989).

[19] S. Sen, F. Capasso, A.Y. Cho, D.L. Sirco, IEEE Trans. Electron. Devices ED- 34, 2185 (1987).

[20] R.C. Potter, A.A. Lakhani, D. Beyea, II.S. Hier, E. Hemplling, A. Fahtimulla, Appl. Phys. Lett. 52, 2163 (1988).

[21] A.A. Lakhani, R.C. Potter, H.S. Hier, Electron. Lett. 24, 681 (1988).

[22] F. Capasso, S. Sen, F. Beltram, A.Y. Cho, in: Physics of Quantum Electron Devices, Ed. F. Capasso, Springer Series in Electronics and Photonics, Vol. 28, Springer, Berlin, Heidelberg, New York 1990, p. 2.

[23] S. Luryi, in: Physics, Fabrication and Applications of Multilayered Structures, Eds. P. Dhez, C. Weisbuch, NATO ASI Series Vol. B 182, Plenum Press, New York, London 1988, p. 241.

[21] A.N. Korotkov, R.H. Chen, K.K. Likharev, J. Appl. Phys. 78, 2520 (1995).

[25] R.H. Chen, A.N. Korothkov, K.K. Likharev, Appl. Phys. Lett. 68, 1954 (1996). 